\title{
An Integrated Road Construction and Resource Planning Approach to the Evacuation of Victims From Single Source to Multiple Destinations
}

\author{
J. W. Wang, W. H. Ip, and W. J. Zhang
}

\begin{abstract}
This paper presents our study on the emergency resource-planning problem, particularly on the development of a new approach to resource planning through contraflow techniques with consideration of the repair of damaged infrastructures. The contraflow technique is aimed at reversing traffic flows in one or more inbound lanes of a divided highway for the outbound direction. As opposed to the current literature, our approach has the following salient points: 1) simultaneous consideration of contraflow and repair of repair of roads; 2 ) classification of victims in terms of their problems and urgency in sending them to a safe place or place to be treated; and 3) consideration of multiple destinations for victims. A simulated experiment is also described by comparing our approach with some variations of our approach. The experimental results show that our approach can lead to a reduction in evacuation time by more than $50 \%$, as opposed to the original resource operation on the damaged transportation network, and by about $20 \%$, as opposed to the approach with resource replanning (only) on the damaged network. In addition, the multiobjective optimization algorithm to solve our model can be generalized to other network resource-planning problems under infrastructure damage.
\end{abstract}

Index Terms-Evacuation, resource planning, road construction, transportation system.

\section{INTRODUCTION}

\section{A. Background and Motivation}

Modern transportation systems are networked systems with high sophistication. Highly sophisticated networking can cause problems, despite the great benefits that they can bring to humanity. One of the problems with such transportation systems is that they tend to be vulnerable when, in particular, the system is

Manuscript received October 6, 2008; revised August 13, 2009; accepted December 3, 2009. Date of publication February 8, 2010; date of current version May 25, 2010. The work of W. J. Zhang was supported in part by the Natural Sciences and Engineering Research Council of Canada and Public Safety and Emergency Preparedness Canada through a joint critical infrastructure program and in part by the National Natural Science Foundation of China under Grant 70931001, Grant 60821063, and Grant 70771021. This work was also supported in part by the Research Fund for the Doctoral Program of Higher Education of China. The Associate Editor for this paper was D. Zeng.

J. W. Wang is with the Department of Mechanical Engineering, University of Saskatchewan, Saskatoon, SK S7N5A9, Canada, and also with the Institute of Systems Engineering, Northeastern University, Shenyang 110004, China (e-mail: juw623@mail.usask.ca).

W. H. Ip is with the Department of Industrial and Systems Engineering, Hong Kong Polytechnic University, Kowloon, Hong Kong (e-mail: mfwhip@ inet.polyu.edu.hk).

W. J. Zhang is with the Department of Mechanical Engineering, University of Saskatchewan, Saskatoon, SK S7N5A9, Canada (e-mail: Chris.Zhang@ Usask.Ca).

Digital Object Identifier 10.1109/TITS.2010.2040276 subject to incidents or accidents. It is then reasonable to assume that in an emergency situation, roads may be damaged. In this paper, we discuss the resource-planning problem for emergency evacuation of victims under situations where roads are partially damaged. More specifically, we consider a scenario that is described by the following features: 1) There are human victims with different categories in an area; 2) these victims need to be evacuated to safe places, respectively, i.e., some to safe place A and other to safe place B; and 3) a network transportation system is damaged to a certain degree due to earthquake, hurricane, or any other cause, and the time to repair the damaged system may be within the length of evacuation time.

The above scenario is practical based on our own experience and observations. For example, a victim that needs CT scan fails to be sent to the CT scan equipment center $2 \mathrm{~h}$ late, and the victim actually dies. In the blizzard in Saskatoon, SK, Canada, in 2006, the recovery of the road around Central Avenue and Preston, which was in fact the only one, played an important role in the evacuation of victims.

One salient point in our approach to emergency evacuation in the above scenario is that we will consider both construction of new roads or the repair of damaged roads and the rerouting of resources to transport victims to safe places, as opposed to the current literature where resource planning and infrastructure building are generally separately conducted. We call our approach an integrated approach to road construction and resource planning to emergency evacuation.

Emergency evacuation in a public transportation system may be relatively new. Therefore, in Section I-B, we will first give a description or definition of the transportation system in light of our purpose.

\section{B. Transportation Systems}

We view a transportation system as having the following two levels: 1) the infrastructure level and 2) the substance or object level; in particular the substance level includes the substance that "flows" over the infrastructure. We view them as the subsystems of an entire transportation system, namely, the transportation infrastructure (TI) system and transportation substance (TS) system. They are, however, related to each other by the constraint that the TS system depends on the TI system or that the TS must "flow" within the TI system. The flow of TS follows certain constraints, which are called "traffic rules." The dynamics of the transportation system are determined by the flow of TS under the constraints of these rules. 


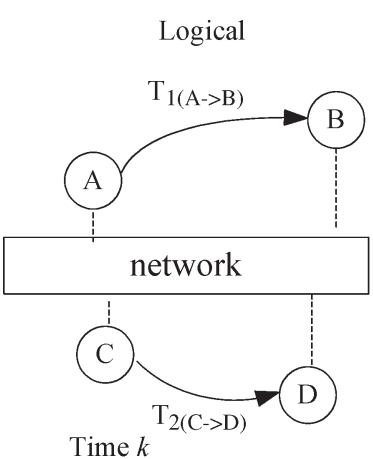

(a)

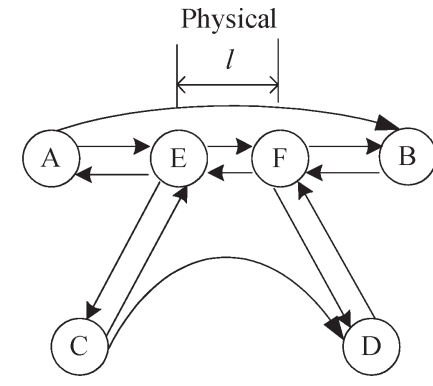

(b)
Fig. 1. Transportation system.

The transportation system is a service system that has the notions of demand and supply. The demand can be generally defined as moving a part of $\operatorname{TS}_{i}(i=1,2, \ldots, n ; n$ is the number of $\mathrm{TS}_{i}$ )'s from one place $(\mathrm{A})$ to another place $(\mathrm{B})$ within a time denoted by $T_{i(\mathrm{~A} \rightarrow \mathrm{B})}$ [see Fig. 1(a)]. Places A and B are a part of a transportation system, particularly its TI system. Further, at one time, e.g., $t$, the demand may be more than one [see Fig. 1(a), where there is a demand from place $\mathrm{C}$ to place $\mathrm{D}$ at time $t$ along with the demand from place $\mathrm{A}$ to $\mathrm{B}$ ]. In Fig. 1(a), we show a logical level of the transportation system at time $t$, and in Fig. 1(b), we show a possible scenario at the physical infrastructure level where the two transportation tasks (A to D; A to B) may share one segment ( $E$ to $F$ ) in the transportation system. Such sharing essentially means that planning the movement of substances becomes extremely difficult. In this paper, the result of resource planning in an emergency situation is a set of TSs, which is denoted by $\{\mathrm{TS}\}$. Sometimes, it is also called a flow pattern.

The supply of service is made with resources, such as vehicles, and infrastructures, such as roads. One of the roadconstruction issues related to infrastructure is road network structure (or configuration of roads). In this paper, the result of road construction is a set of TIs, which is denoted by $\{\mathrm{TI}\}$.

In a normal situation, the configuration of infrastructure is fixed, i.e., the road is constructed first, and then, vehicles are put into use on the road. In a normal traffic situation, the road configuration construction and resource planning are two separate activities; in particular, resource planning is done at the stage where the road is in use or in operation. However, in an emergency situation or in a situation where the TI is partially damaged (e.g., some roads are damaged in disasters and are no longer available for mass transportation), the road construction and resource planning may turn out to be integrated (i.e., two activities of the determination of $\{\mathrm{TI}\}$ and $\{\mathrm{TS}\}$ take place simultaneously).

For example, when a component of the transportation system is damaged, we need to consider whether the "maximum" capacity of the remaining TIs can still satisfy the demand. If not, we have to construct the road infrastructure (i.e., adding new roads or repairing the damaged roads), namely, changing the $\{\mathrm{TI}\}$. It is also possible that through resource replanning on the remaining system, namely, changing the $\{\mathrm{TS}\}$, the demand can simply be satisfied. Further, it may be the case that both road construction and resource planning are considered together

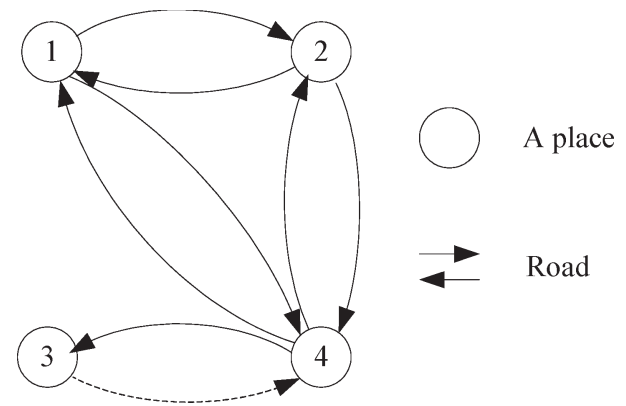

Fig. 2. Conceptual model of the transportation system (place, road, and lane).

(i.e., on one hand, we add new roads or repair damaged roads, while on the other hand, we replan the resources). This last case is aimed at changing $\{\mathrm{TI}\}$ and $\{\mathrm{TS}\}$ simultaneously, which makes sense in the so-called integrated approach to emergency evacuation. Hereafter, in this paper, this approach is termed the TI-TS emergency evacuation.

\section{Objectives of Our Study and Organization of This Paper}

In the study presented in this paper, our objective was to develop a model that can allow representing the following: 1) the integrated road reconstruction and resource-planning strategy for emergency evacuation (TI-TS emergency evacuation) of victims from one single place to multiple destinations according to a victim's special requirement; 2) different categories of victims with different evacuation demands; and 3) different optimal flow patterns for different categories of victims with the contraflow technique.

The remainder of this paper is organized as follows. Section II will present a conceptual model of our problem. In Section III, we present a mathematical model, followed by a detailed discussion of a three-layer method to solve this model. It is noted that according to Kim and Shekhar [1], the planning problem here is an NP-complete problem. Therefore, our algorithm for planning (second layer in the whole method) is an intelligent evolutionary computing algorithm, namely, particle swarm optimization (PSO). Section V presents an example to demonstrate the effectiveness of our approach. Section VI gives some discussion of related work. Section VII presents a conclusion with some discussion.

\section{Conceptual Model of the Problem}

\section{A. Transportation System}

In the problem that we consider here, there is one place in a dangerous area (single source) where there are a few categories of victims who are to be evacuated. There are also a few different categories of safe places to which the victims are to be sent. Our problem can be called a "single-source-multipledestinations" (SSMD) problem. There is a traffic network between the source and the destinations. The traffic network system is composed of a set of places and a set of roads (see Fig. 2). Each place has a capacity and an initial occupancy. The capacity of a place is defined as the maximum number of people who can stay in the place. The initial occupancy of a place is the number of residences who are to be evacuated. Each road 


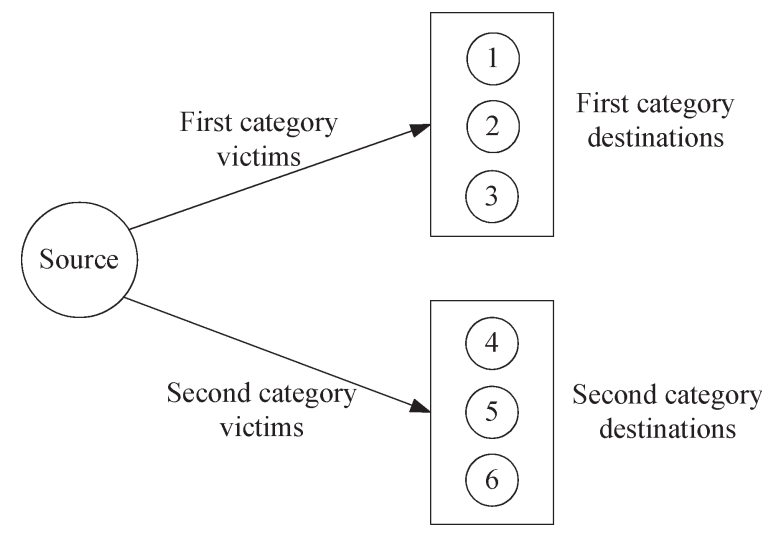

Fig. 3. Demands of victims of different categories.

has lanes that have either directions and are grouped in terms of their directions, i.e., there are two groups. Each group of lanes forms one edge in the context of graph or network. In Fig. 2, edge $1 \rightarrow 2$ is a group of lanes with the same direction in road 1-2, and edge $2 \rightarrow 1$ is another group of lanes with the opposite direction in road 1-2. We further consider the case that a road has lanes that have all in one direction as a special case of the general case that a road has lanes that have opposite directions, e.g., road 3-4 in Fig. 2. Each lane has a capacity and a travel time. The capacity of a lane is defined as the number of evacuees per unit period, which is prescribed or given. Each edge also has a capacity and a travel time. The capacity of an edge is the sum of those of the lanes in this edge. The travel time of an edge is equal to the travel time of each lane in this edge.

\section{B. Evacuation Demands}

The victims on the source place are ranged into different categories based on their characteristics. The destination places are also classified into different categories according to the categories of the victims that they receive. We label the different categories of victims with different evacuation priorities. Different categories of victims have different evacuation demands, as shown in Fig. 3: 1) The victims of the same category will be evacuated to the corresponding destinations; for instance, the victims who are injured need to be sent to the hospital, and the victims without injury need to be sent to their places of residence, and 2) the victims with higher priority will be evacuated earlier, e.g., the injured victims need to be evacuated first.

\section{Integrated Road Constraction and Resource Planning: TS-TI}

In this paper, we consider the road reconstruction along with the resource replanning. Further, a road is associated with the following attributes: 1) edge capacity; 2) lane capacity; and 3) place capacity. We consider the reconstruction of roads as restricted by the following conditions: 1) Only one lane can be added into a remaining damaged transportation network; 2 ) the new lane $i \rightarrow j$ falls into the edge domain of the original transportation network; 3 ) the travel time of the new lane $i \rightarrow j$ is the same as the original travel time $t_{i j}$ of the lane $i \rightarrow j$ in the original transportation network; and 4) the capacity of the

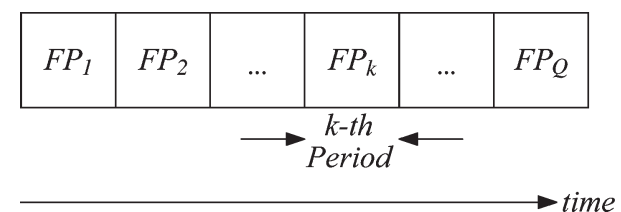

Fig. 4. Dynamic flow patterns during the whole evacuation process.
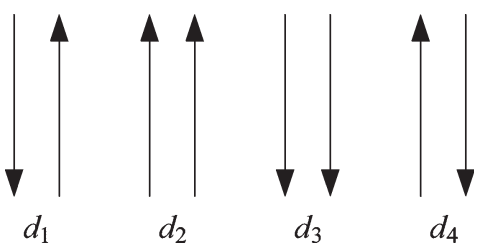

Fig. 5. Flow patterns on a single road.

new lane is a constant (this constant can be different from those for other lanes).

The resource planning of the transportation system is meant to determine such parameters as travel time and the number of lanes in one direction for a particular road. Our problem here is to plan the flow pattern of the transportation network. To meet the demand to transport the different categories of victims, we need to plan the flow pattern first for the victims of the first category, then for the victims of the second category, and so on. If there are $Q$ categories of victims, the planning of the flow pattern will be divided into $Q$ periods. During the $k$ th period, the $k$ th category has the highest priority to use the transportation network; furthermore, the other categories will be evacuated concurrently when there is extra transportation capacity. In this case, there will be $Q$ flow patterns of the transportation network during the evacuation process, as shown in Fig. 4. Usually, the best flow patterns for the victims of different categories are different, and hence, the flow pattern planning is dynamic. The setup time of the flow pattern is not considered here for simplicity.

It is worth stressing the point that road reconstruction only takes place in disaster situations where, in particular, a road network system is partially damaged. The situation of the intact road network is considered as a special case of the general situation we consider here. Furthermore, a need to construct new roads also arises due to a sudden increase in transportation demands in a particular region or regions such that the existing TI is over its capacity.

Furthermore, the road reconstruction and resourcereplanning activities must be carried out concurrently to render a transportation performance that is both optimal and feasible. The optimal performance means that the cost of a proposed construction and planning is minimal, while the feasible performance means that a particular plan (or particular TS system) can be realized by a given physical structure of the transportation system (or a given TI system).

\section{Mathematical Model of the Problem}

We represent the state of road in terms of edges, as shown in Fig. 5. In this figure, $d_{1}, d_{2}, d_{3}$, and $d_{4}$ represent four states with corresponding edge patterns associated with each of them. For the $k$ th $(1 \leq k \leq Q)$ flow pattern, we add the information 


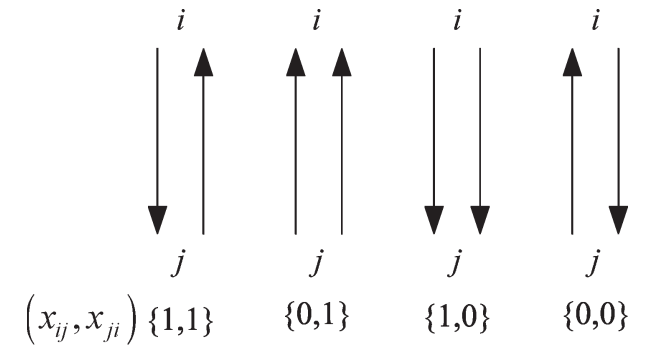

Fig. 6. Domain of the variable to represent the flow patterns on a single road.

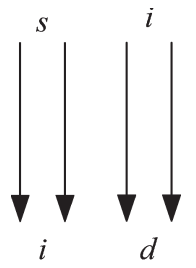

(a) (b)

Fig. 7. Flow patterns that are associated with source place and destination place.

of the place (i.e., $i, j$ ) to Fig. 5, resulting in Fig. 6. We introduce variable $x_{i j}^{k}$ to represent the information of edge $i \rightarrow j$ during the $k$ th period; in particular, $x_{i j}^{k}=1$ represents the flow from $i$ to $j$, and $x_{i j}^{k}=0$ represents the flow from $j$ to $i$. As such, the state of road connecting place $i$ and place $j$ during the $k$ th period can be expressed by $\left(x_{i j}^{k}, x_{j i}^{k}\right)$; in particular, the four states of the road are represented by $\left(x_{i j}^{k}, x_{j i}^{k}\right)$, as shown in Fig. 6. It should be noted that when the travel times and capacities of the two edges in one road are different, the pattern $d_{1}$ and the pattern $d_{4}$ are different. Only when in the special case that the travel times and capacities of the two edges in one road are the same, the pattern $d_{1}$ and the pattern $d_{4}$ have the same transportation ability. This has implied the need for the consideration of the four flow patterns (instead of three patterns considered by Kim and Shekhar [1]) in an emergency situation.

For the evacuation of victims from a source place $s$ to a destination place $d$, the flow pattern of the roads associated with these two nodes should be ones that are shown in Fig. 7. In this figure, we show that for the source place, all flows must be outgoing, and for the destination node, all flow must be incoming. It should be noted that when we plan the flow pattern for the victims of the first category, only the destinations of the first category are regarded as the destinations, and the destinations for the victims of the other categories are regarded as the common places.

Let us use "node" for place and "arc" for edge. A particular transportation system has $m$ nodes and $n$ directed arcs. As such, a transportation system can be represented as a directed graph denoted by $G$ and $G=(N, A)$. A flow pattern or configuration is defined as a snapshot of the network $G$, starting from the transportation or evacuation task to the completion of the task. Each node has a capacity and an initial occupancy. Each arc also has a capacity and a constant travel time. Next, we will give definitions about the evacuation time in our problem.

The individual evacuation time of one evacuee $\left(E T_{i}\right.$, where $i$ stands for this evacuee) is defined as a period of time from

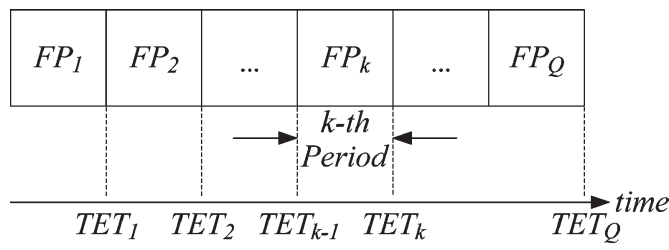

Fig. 8. Dynamic flow patterns with horizontal coordinate.

the moment when the first evacuee in the crowd leaves from a source node to the moment when evacuee $i$ arrives at a destination node.

The total evacuation time of the $k$ th category victims $\left(T E T_{k}\right)$ is defined as a period of time from the moment when the first evacuee of the first category leaves from a source node to the moment when the last evacuee of the $k$ th category arrives at a destination node. The mathematical expression of $T E T_{k}$ will be given in Section III-B.

The average evacuation time of $k$ th category $\left(A E T_{k}\right)$ is defined as

$$
\mathrm{ATE}=\frac{\sum_{i=1}^{q} E T_{i}}{q}
$$

where $q_{k}$ is the total number of evacuees of the $k$ th category on the source node. Based on the definition of $A E T_{k}$, we can get the average evacuation time $(A E T)$ of the victims of all the categories as

$$
A E T=\frac{1}{\sum_{k=1}^{Q} q_{k}} \sum_{k=1}^{Q} \sum_{i=1}^{q_{k}} E T_{i} / q_{k} .
$$

Based on the definitions above, we can add a horizontal coordinate to Fig. 4, resulting in Fig. 8.

Assume that at the beginning, there are several categories of victims on a certain node. With consideration of the priorities of different categories of victims, the objective of evacuation planning is to find flow patterns to minimize the total evacuation time of the $k$ th category victims $(k=1,2, \ldots, Q)$, i.e., $T E T_{k}$, based on minimizing the $T E T_{k-1}$.

\section{A. Variable Definition}

$G=(N, A)$

directed network with $N$ being the set of nodes and $A$ being the set of arcs (static network);

$m=|N| \quad$ number of elements in set $N$;

$n=|A|$

$c_{i j}$

$\lambda_{i j}$ number of elements in set $A$;

capacity of arc $(i, j)$;

travel time of arc $(i, j), \forall(i, j) \in A$;

node capacity, number of evacuees that can stay in node $i$;

source node;

category number of evacuees;

initial number of evacuees of $k$ th category in node $s, k=1, \ldots, Q$;

$\operatorname{pred}(i) \quad=\{j \mid(j, i) \in A\}$; predecessors of node $i$; $\operatorname{succ}(i) \quad=\{j \mid(i, j) \in A\}$; successors of node $i$; 


\begin{tabular}{|c|c|}
\hline$D_{k}$ & set of destinations that receive the evacuees \\
\hline & of $k$ th category, $k=1,2, \ldots, Q$ \\
\hline & set of destinations, $D_{k} \subset D, k=1,2, \ldots, Q$; \\
\hline$G_{T}=\left(N_{T}, A_{T}\right)$ & $\begin{array}{l}\text { time expansion of } G(N, A) \text { over a time } \\
\text { horizon } T \text {, where } T \text { is the predetermined } \\
\text { upper bound of the total travel time; }\end{array}$ \\
\hline & $=\{i(t) \mid i \in N ; t=0,1, \ldots, T\}$ \\
\hline & set of arcs over a time horizon $T$; \\
\hline & super destination node; \\
\hline & $\begin{array}{l}\text { flow (number of evacuees) of } k \text { th category } \\
\text { that leave node } i \text { at time } t \text { and reach node } j \\
\text { at time } t+\lambda_{i j} \forall i, j \in N, k=1, \ldots, Q ;\end{array}$ \\
\hline$y_{i}(t+1)$ & $\begin{array}{l}=\sum_{k=1}^{Q} f_{i(t), i(t+1), k} \forall i \in N, t=0, \ldots, \\
T: \text { number of evacuees who prefer to stay } \\
\text { in the node } i \text { at time } t \text { for at least one unit } \\
\text { time; }\end{array}$ \\
\hline & $\begin{array}{l}\text { state of flow from } i \text { to } j \text { on the } \operatorname{arc}(i, j) \\
\text { during the } k \text { th period, } k=1, \ldots, Q ;\end{array}$ \\
\hline & $\begin{array}{l}\text { flow pattern between the node } i \text { and } j \text { dur- } \\
\text { ing the } k \text { th period, } k=1, \ldots, Q \text {; }\end{array}$ \\
\hline
\end{tabular}

$F(k)$

$z_{i j}^{k}$

Cadd

The decision variables of this model are $F(k)$ and $z_{i j}^{k}$.

\section{B. Objective Function and Constraints}

\section{SOLVING THE Model}

Our proposed method of solving the model [see (3)-(18), shown at the bottom of the page] is an algorithm with three layers (i.e., upper layer algorithm, middle-layer algorithm, and lower layer algorithm), which are further coupled, as shown in Fig. 9. In each round of iteration, the upper layer algorithm will need to call the middle-layer algorithm, and the middlelayer algorithm will need to call the lower layer algorithm. The
Equation

$$
\begin{aligned}
& \text { Min } \quad\left\{T E T_{k} \mid \min T E T_{k-1}, k=2, \cdots, Q\right\} \\
& \text { s.t. } \quad T E T_{k}=\left\{T \mid f_{i j k}\left(T^{\prime}\right)=0, \forall T^{\prime}>T\right\} \\
& x_{i j}^{k}=0,1 \forall(i, j) \in A, k=1, \ldots, Q \\
& \bar{x}_{i j}^{k}=\left\{\begin{array}{ll}
0, & x_{i j}^{k}=1 \\
1, & x_{i j}^{k}=0
\end{array}, \forall(i, j) \in A, k=1, \ldots, Q\right. \\
& z_{i j}^{k}=0,1 \forall(i, j) \in A, k=1, \ldots, Q \\
& \bar{z}_{i j}^{k}=\left\{\begin{array}{ll}
0, & z_{i j}^{k}=1 \\
1, & z_{i j}^{k}=0
\end{array}, \forall(i, j) \in A, k=1, \ldots, Q\right. \\
& \sum_{\forall(i, j) \in A} z_{i j}^{k}=1, k=1, \ldots, Q \\
& \sum_{t=0}^{T} \sum_{i \in \operatorname{succ}(s)} f_{s i k}(t)=q_{k}, k=1, \ldots, Q \\
& \sum_{t=0}^{T} \sum_{i \in D_{k}} f_{i d k}(t)=q_{k}, k=1,2, \ldots, Q \\
& y_{i}(t+1)-y_{i}(t)=\sum_{l \in \operatorname{pred}(i)} \sum_{k=1}^{Q} f_{l i k}\left(t-\lambda_{l i}\right) \\
& -\sum_{j \in \operatorname{succ}(i)} \sum_{k=1}^{Q} f_{i j k}(t), \forall i \in N, t=0, \ldots, T \\
& y_{i}(0)=0, \forall i \in N, i \neq s \\
& y_{i}(t)=0, \forall i \in D, t=0, \ldots, T \\
& 0 \leq y_{i}(t) \leq a_{i}, t=1, \ldots, T, \forall i \in N-D \\
& 0 \leq \sum_{k=1}^{Q} f_{i j k}(t) \leq x_{i j}^{k}\left(c_{i j}+z_{i j}^{k} c_{a d d}\right)+\bar{x}_{j i}^{k}\left(c_{j i}+\bar{z}_{i j}^{k} c_{a d d}\right), \\
& t=0, \ldots, T E T_{k}-\lambda_{i j}, \forall(i, j) \in A, k=1, \ldots, Q \\
& x_{s i}^{k}=1, x_{i s}^{k}=0, \forall(s, i) \in A, k=1, \ldots, Q \\
& x_{i j}^{k}=1, x_{j i}^{k}=0, \forall(i, j) \in D_{k}, k=1, \cdots, Q
\end{aligned}
$$

Description

(3) objective function

(4) total evacuation time of the $k$-th category

(5) $x_{i j}^{k}$ is a 0 - 1 variable

(6) definition of $\bar{x}_{i j}^{k}$

(7) $z_{i j}^{k}$ is a $0-1$ variable

(8) definition of $\bar{z}_{i j}^{k}$

(9) restriction of the number of new lanes.

Initial victim numbers

$D_{k}$ only receives $k$-th category

flow conservation constraint

no victims on the nodes, except $s$

no victims on $D_{k}$

node capacity constaint

arc capacity constraint

flow pattern of the roads linked with $s$

flow pattern of the roads linked with $D_{k}$ 


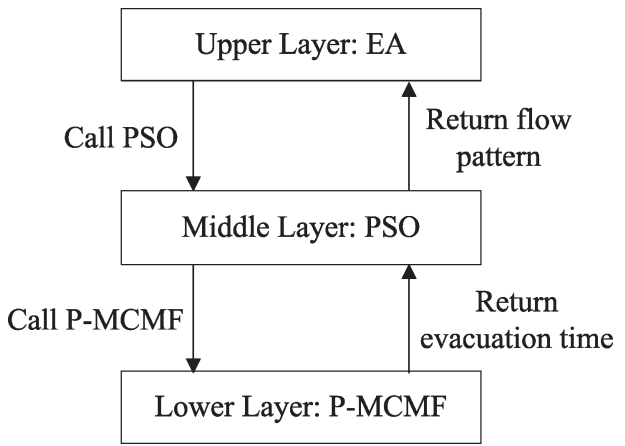

Fig. 9. Sketch of the three-layer algorithm.

lower layer algorithm, which is called priority-based minimum cost multicommodity flow (P-MCMF) algorithm, is aimed at seeking the minimum flow time for multicommodities given a flow pattern. The middle-layer algorithm takes an evolutionarycomputing algorithm, which is called PSO, to find the best flow pattern in a given designed transportation network. The upper layer algorithm is aimed at finding the best design result for the damaged transportation network, and it takes an enumeration algorithm (EA) since the solution space is limited. The threelayer algorithm will be discussed in detail in the following sections.

\section{A. Lower Layer Algorithm}

Our problem is, in essence, a problem of P-MCMF. This problem has been studied in the operations research area, and there are many algorithms that have been developed [2], e.g., the basis partitioning method [3], the resource-directive method [4], the price-directive method [5], the primal-dual method [6], the approximation method [7], the interior-point method [8], the convex programming method [9], and heuristics [10]. However, the following two issues remain if these algorithms are used for our model: 1) The existing MCMF algorithm is designed for a static network with no restriction of the node capacity, and 2 ) the existing MCMF algorithm is designed with no consideration of priorities.

The first issue can easily be resolved by converting the original traffic network into a time-expanded network (TEN). For the second issue, because there are different priorities for different kinds of people and the flow pattern cannot be changed during one period, the flow-pattern optimization has to be taken as a multiperiod problem. During one period, the optimization of the evacuation time is similar with the minimum cost flow problem. Nevertheless, we still cannot use any existing minimum cost flow algorithm directly as the lower layer algorithm for the following reasons: 1) More than one category of victims may need to be evacuated to some safe destinations, and 2) the victims of different categories have different priorities. Therefore, we designed a P-MCMF algorithm as the lower layer algorithm.

The main ideas of the P-MCMF algorithm are given as follows: 1) During the $k$ th period of the evacuation process, evacuate all the $k$ th category victims as soon as possible, which has the same function as any existing minimum cost flow algorithm; 2) at the same time, the $k^{\prime}$ th $\left(k^{\prime}=k+1, \ldots, Q\right)$ categories are evacuated as much as possible. This second idea further means that the transportation network may have

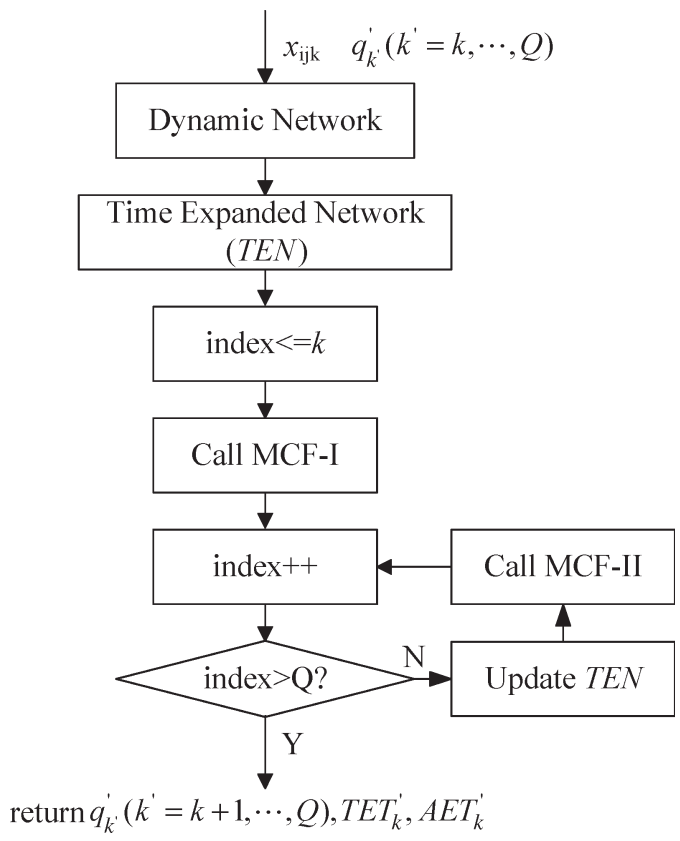

Fig. 10. Flowchart of the P-MCMF algorithm.

an ability to evacuate $k^{\prime}$ th $\left(k^{\prime}=k+1, \ldots, Q\right)$ categories of victims when evacuating the $k$ th category victim. The details of the P-MCMF algorithm are expressed in Fig. 10.

From the lower layer algorithm, we can get $T E T_{k}^{\prime}, A E T_{k}^{\prime}$, and $q_{k^{\prime}}^{\prime}\left(k^{\prime}=k+1, \ldots, Q\right)$. Here, $q_{k^{\prime}}^{\prime}$ refers to the number of $k^{\prime}$ th category $\left(k^{\prime}=k+1, \ldots, Q\right)$ victims that have not been evacuated after the $k$ th period. It is obvious that $q_{k}^{\prime}$ is not larger than $q_{k}$, and $T E T_{k}^{\prime}$ can be expressed by

$$
T E T_{k}^{\prime}=T E T_{k}-T E T_{k-1} .
$$

$A E T_{k}^{\prime}$ refers to the $A E T$ of the $q_{k}^{\prime}$ victims of the $k$ th category, which is evacuated from $T E T_{k-1}$ to $T E T_{k}$. The block "Update TEN" has the following two operations: 1) updating the capacity matrix and 2) updating the destination nodes.

It should be noted that MCF-I and MCF-II are different. MCF-I is an existing classic minimum-cost-flow algorithm, e.g., the dual ascent algorithm, which is used to evacuate the $k$ th category victims whose number is $q_{k}^{\prime}$ and get the evacuation time, including $A E T$ and TET. MCF-II is used to calculate the numbers of victims of $k^{\prime}$ th category $\left(k^{\prime}=k+1, \ldots, Q\right)$ that can be evacuated during the $k$ th period. The differences between MCF-I and MCF-II are listed as follows: 1) There is a vector in MCF-II, which is labeled as $f_{\text {exist }}$, to record the flows that have been on the transportation network, which means that the capacity of the edges decreases, and 2) the maximum time in MCF-II, namely, the number of time units of the TEN, is fixed, which is equal to TET derived by MCF-I, and MCF-II is used to calculate the number of victims of the $k^{\prime}$ th category $\left(k^{\prime}=\right.$ $k+1, \ldots, Q)$ that can be evacuated within the maximum time.

\section{B. Middle-Layer Algorithm}

As mentioned in Section I-C, the planning problem in this model is an NP-complete problem. As a result, intelligent optimization algorithms, such as genetic algorithm (GA), simulated 
annealing (SA), Tabu search (TS), and PSO, are potential options as the middle-layer algorithm. Furthermore, the fitness value of the middle algorithm, namely, the minimum evacuation time, must be obtained by calling the lower layer algorithm, which will cost much running time. To our previous experience, GA is relatively slow in getting the global optimum. SA and TS, as individual-based algorithms, trend to get into the local optimum. Therefore, we choose the PSO algorithm to get a tradeoff between exploration and exploitation in this problem.

The PSO algorithm was first developed by Kennedy and Eberhart based on the simulation of a simplified social model [11]-[13]. The algorithm has attracted increasingly more attention [14]-[17]. The standard PSO algorithm can be explained as follows.

A swarm being made up by $m$ particles searches a $D$-dimensional problem space. Each particle is assigned a randomized velocity and a stochastic position. The position represents the solution to the problem: when each "flying" particle is attracted by a good location achieved so far by itself and by a good location achieved by the members in the whole swarm (or the members in the neighborhood). The position of the $i$ th particle is represented as $x_{i}=\left(x_{i 1}, \ldots, x_{i d}, \ldots, x_{i D}\right)$, and its velocity is represented as $v_{i}=\left(v_{i 1}, \ldots, v_{i d}, \ldots, v_{i D}\right)$, $1 \leq i \leq m, 1 \leq d \leq D$. The best previous position of the $i$ th particle, namely, the position with the best fitness value, is represented as $p_{i}=\left(p_{i 1}, \ldots, p_{i d}, \ldots, p_{i D}\right)$, and the index of the best particle among all the particles in the population (or in the neighborhood) is represented by the symbol $g$. Each particle updates its velocity and position according to the following equations:

$$
\begin{aligned}
& v_{i d}^{k+1}=\omega v_{i d}^{k}+c_{1} \xi\left(p_{i d}^{k}-x_{i d}^{k}\right)+c_{2} \eta\left(p_{g d}^{k}-x_{i d}^{k}\right) \\
& x_{i d}^{k+1}=x_{i d}^{k}+v_{i d}^{k+1}
\end{aligned}
$$

where $\omega$ is the inertia weight that determines how much current velocity that a particle holds in the next iteration. A suitable selection of inertia weight can provide the particles with a balance between the ability of exploitation and exploration. $c_{1}$ and $c_{2}$ are learning factors, which are also called acceleration constants, which are two positive constants. Learning factors are usually equal to 2 , while other settings can also be seen in the literature [15]. $\xi$ and $\eta$ are pseudorandom numbers, and they obey the same homogeneous distribution in the range $[0,1]$. The velocity of a particle is limited in the range of $V_{\max }$. $V_{\max }$ is set to be the range of each dimension variable and is used to initialize the velocity of particles without selecting and tuning in detail in the experiments. The running of the PSO is quite similar to evolutionary algorithms such as GA, including initialization, fitness evaluation, update of velocity and position, and testing of the stop criterion.

The standard PSO algorithm is for the continuous variableoptimization problem. The PSO algorithm for the discrete variable problem can be found in [17] and [18]. Our problem is a discrete variable problem, and therefore, we apply the discrete PSO algorithm. Details of the PSO for our problem include 1) encoding, 2) update formula, and 3) stop criterion, and they are given as follows.

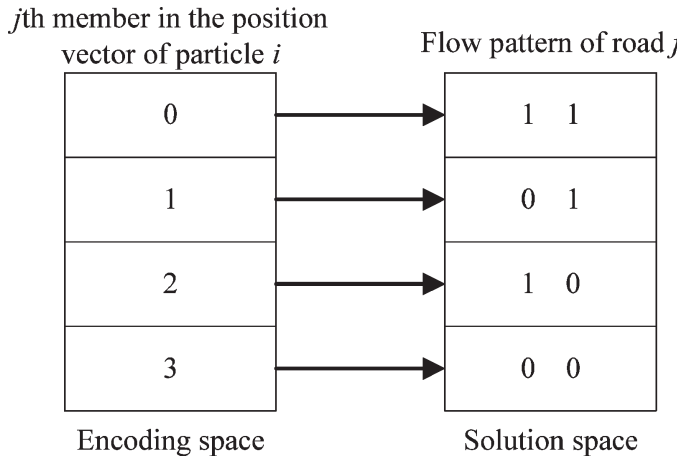

Fig. 11. Conversion between the encoding space and the solution space.

1) Encoding: Encoding is used to design the representation of the position of a particle. Let

$n_{R}$ number of roads in the transportation network;

$n_{s} \quad$ number of roads linked to the source node which is equal to the number of $\operatorname{arcs}(s, i) \forall(s, i) \in A$;

$n_{k} \quad$ number of roads linked to the destination node which is equal to the number of $\operatorname{arcs}(i, j) \forall(i, j) \in A, j \in D_{k}$, $k=1,2, \ldots, Q$.

The number of roads whose flow patterns are unknown is represented as follows:

$$
L=n_{R}-n_{s}-n_{k} .
$$

The position of particle $i$ is represented as

$$
x_{i}=\left(x_{i 1}, \ldots, x_{i j}, \ldots, x_{i L}\right), \quad 1 \leq j \leq L
$$

where $x_{i j} \in\{0,1,2,3\}$, which will be explained further later in this paper.

There is a conversion between the encoding space and the solution space, which is illustrated in Fig. 11. In Fig. 11, the left part is the code, and the right part is the flow pattern of a road. The mapping function is self-explained in the figure.

2) Update Formulas: The position vector of a particle is composed of integers, and therefore, the update formula of the position and the velocity is designed as follows:

$$
\begin{aligned}
& v_{i j}^{k+1}=\left\{\begin{array}{cc}
\operatorname{Int}\left(\omega v_{i j}^{k}+c_{1} \xi\left(p_{i j}^{k}-x_{i j}^{k}\right)\right. \\
\left.+c_{2} \eta\left(p_{g j}^{k}-x_{i j}^{k}\right)\right), & \gamma \leq c_{0} \\
\operatorname{Int}\left(\omega v_{i j}^{k}+\operatorname{Random}(0,4)\right), & r>c_{0}
\end{array}\right. \\
& x_{i d}^{k+1}=\left|x_{i d}^{k}+v_{i d}^{k+1}\right| \bmod (4)
\end{aligned}
$$

where $\operatorname{Int}(\cdot)$ is the function to get the integer part of $(\cdot), c_{1}$ and $c_{2}$ are learning factors, which are two positive constants, $\xi, \eta, \gamma \in U[0,1]$, which are pseudorandom numbers that obey the same homogeneous distribution in the range $[0,1]$, and $c_{0}$ is a constant in $[0,1]$, which is used to decide the update mode of the velocity. The formula in (24) indicates that when a random number $\gamma$ is not greater than $c_{0}$, the velocity is updated according to the traditional mode, which is described as follows:

$$
v_{i j}^{k+1}=\operatorname{Int}\left(\omega v_{i j}^{k}+c_{1} \xi\left(p_{i j}^{k}-x_{i j}^{k}\right)+c_{2} \eta\left(p_{g j}^{k}-x_{i j}^{k}\right)\right) .
$$


When $\gamma$ is greater than $c_{0}$, the velocity is updated according to the random mode, which is given as follows:

$$
v_{i j}^{k+1}=\operatorname{Int}\left(\omega v_{i j}^{k}+\operatorname{Random}(0,4)\right)
$$

where $\operatorname{Random}(0,4)$ is a random generator between 0 and 4. Further, the mod number is used to prevent the particle from flying out of the boundary. The absolute value of $|\cdot|$ is used to keep the position nonnegative.

3) Stop Criterion: A positive integer $N G$ is taken as the maximum iteration number. If the iteration number is greater than $N G$, the algorithm is stopped. $N G$ is set based on trialand-error for a particular problem.

The entire procedure of the algorithm has the following steps.

Step 1) Set $c_{1}, c_{2}$, and $c_{0}$. Initialize a swarm including $m$ particles with random positions inside the solution space. $x^{*}$ is the best solution, and $f\left(x^{*}\right)$ is the best fitness value.

Step 2) Call the P-MCMF algorithm to evaluate the fitness of each particle. The index of the one that gets the best fitness is set as $g$. The position and fitness of the particle $g$ are used to update $x^{*}$ and $f\left(x^{*}\right)$.

Step 3) Update the velocity and position of particles according to (24) and (25).

Step 4) If the stop criterion is satisfied, the algorithm ends; otherwise, go to Step 2).

\section{Upper Layer Algorithm}

The upper layer algorithm is to find the best design result by adding one lane to some edge in the damaged transportation network. It is obvious that the solution space is limited. Therefore, an EA can be taken as the upper layer algorithm, which is illustrated in Fig. 12.

At the beginning, $A E T_{k}$ and $T E T_{k}$ are set as the biggest integers in a 32-bit computer. The block "Update $A E T_{k}$ and $T E T_{k}$ " is taken according to (19).

\section{EXAMPLE}

\section{A. Problem Description}

Fig. 13 illustrates an original transportation system that has seven places. The capacity of each place is represented by a bracket " \{\} " (see Fig. 13), e.g., the capacity of place 1 is 120. Further in Fig. 13, the "-" in the bracket adjacent to places 6 and 7 means that places 6 and 7 are the shelters (i.e., their capacities are unlimited). The road and edge are expressed by the connection between places (see Fig. 13). Both capacity and travel time of the edge are indicated on the arc by a parenthesis "( )", e.g., edge $1 \rightarrow 2$ has travel time 2 and capacity 1 , and edge $2 \rightarrow 1$ has travel time 2 and capacity 2 (see Fig. 13). The time unit in the example is minute, which is omitted in the following paragraphs.

Further in this evacuation problem, place 1 is a source node, and places 6 and 7 are two sink nodes. Place 1 has two categories of evacuees, namely, the number of the victims in the first category is 15 , and the number in the second category is 93 .

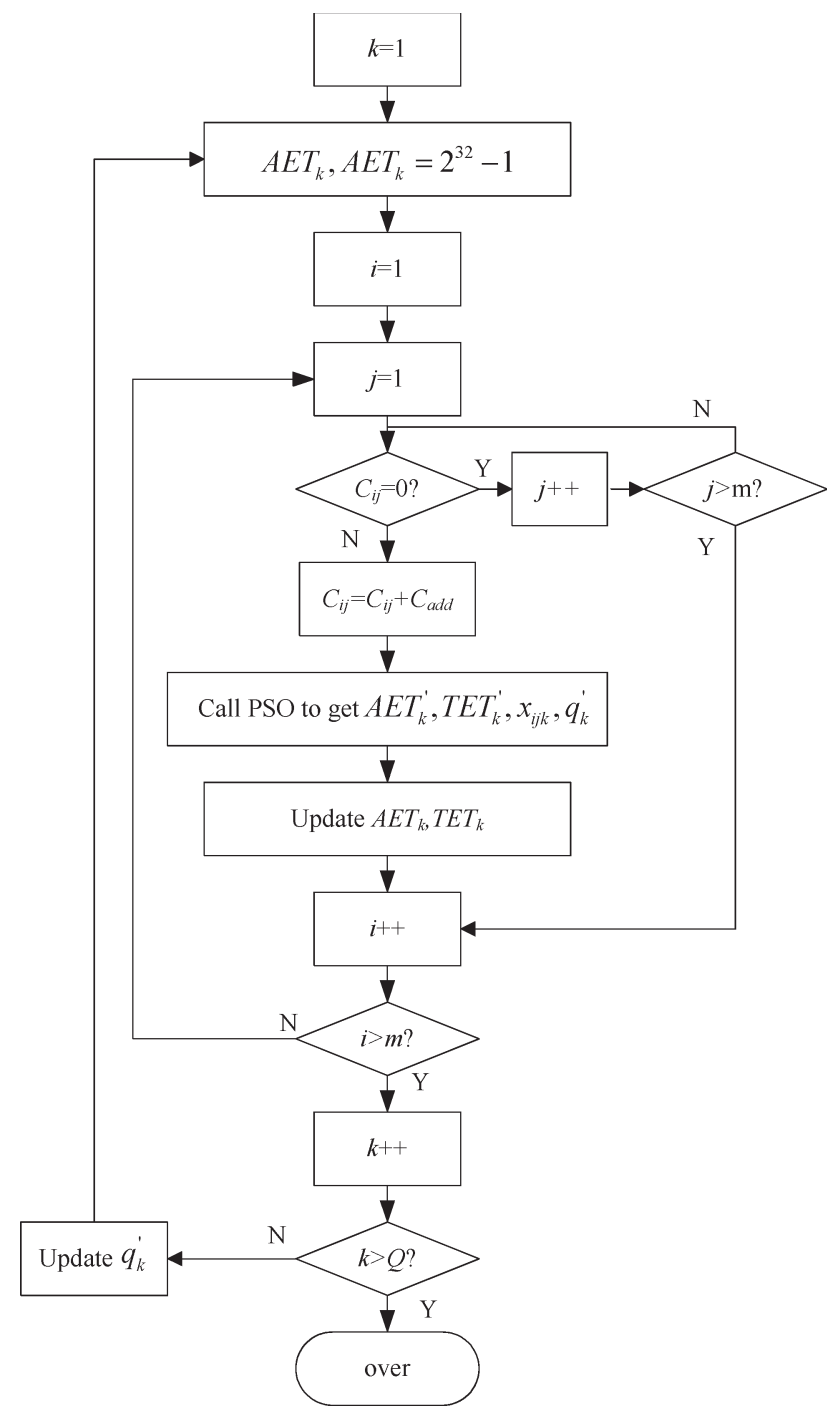

Fig. 12. Flowchart of EA.

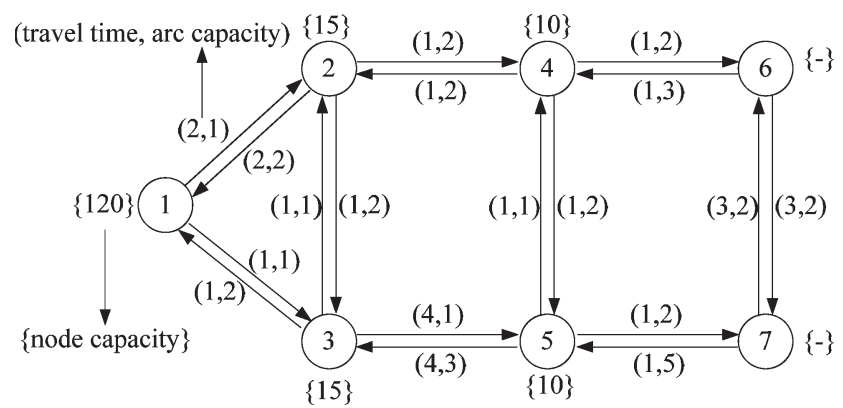

Fig. 13. Original transportation system.

The victims in the first category must be evacuated to place 6 , and the victims in the second category must be evacuated to place 7. Fig. 14 represents the evacuation situation on the transportation network that has been partially damaged by losing the edge $4 \rightarrow 5$.

The design here is to add one lane on the damaged system. The new lane $i \rightarrow j$ falls into the edge domain of the original transportation system, and the travel time of the new lane $i \rightarrow j$ is the same as the original travel time $t_{i j}$ of the edge $i \rightarrow j$ 


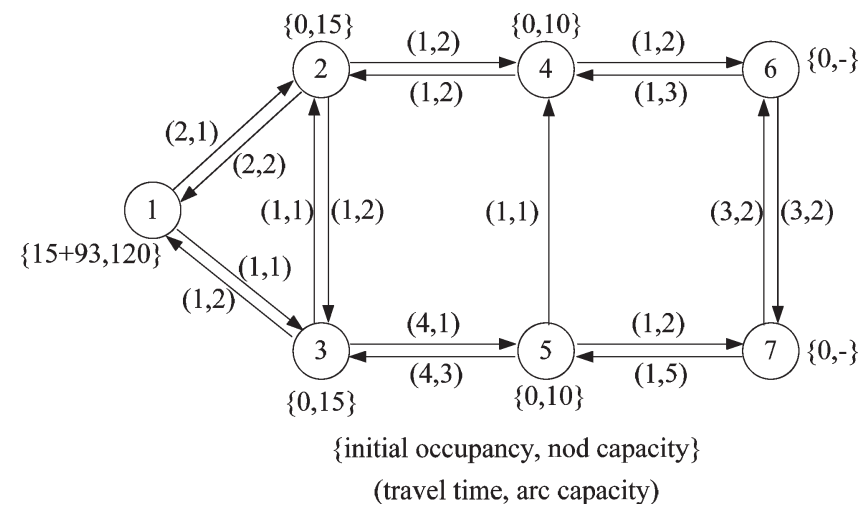

Fig. 14. Damaged transportation system (the source node is 1; the destination node for the first category is 6 and for the second category is 7).

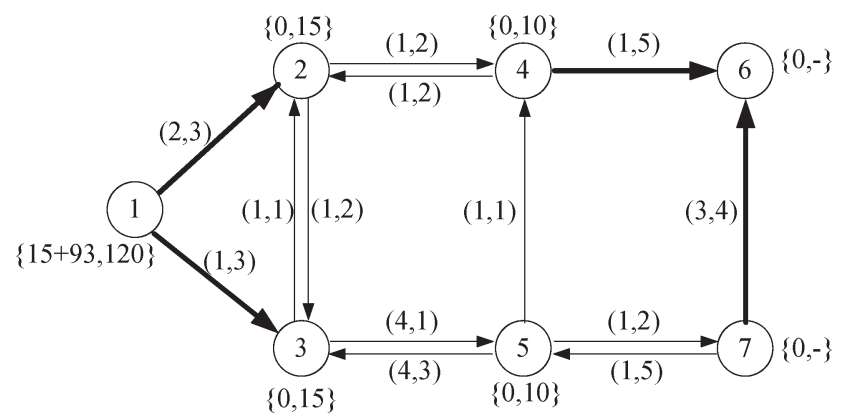

Fig. 15. Predefined transportation network for category 1 on the damaged system described in Fig. 14.

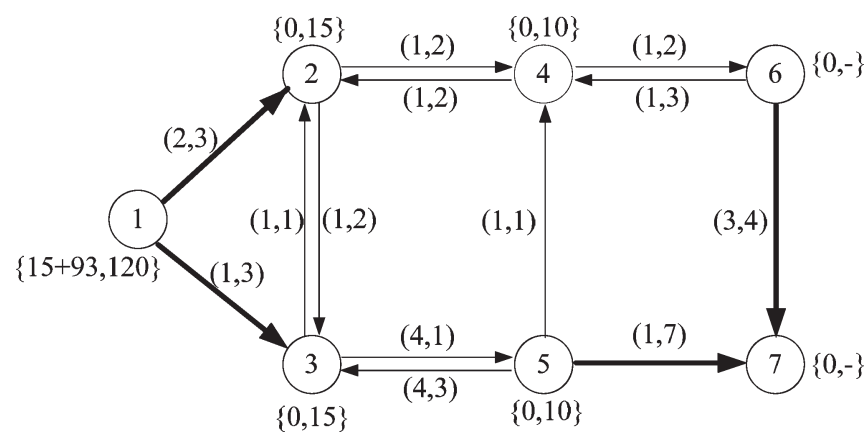

Fig. 16. Predefined transportation network for category 2 on the damaged system described in Fig. 14.

in the original transportation network, as explained in Section II-C. The capacity of the new lane is 2. Further, according to the constraint equations in (16) and (17), we have already known that the flow patterns for edges $(1,2),(1,3)$, $(4,6)$, and $(6,7)$ during the first evacuation period should be ones that are shown in Fig. 15, and the flow patterns for edges $(1,2),(1,3),(5,7)$, and $(6,7)$ during the second evacuation period should be ones that are shown in Fig. 16. This implies that these edges are no longer a decision variable.

In the experiment, we consider the following four different methods of evacuating: 1) damaged transportation network without any modification and planning; 2) predefined networks in which the flow patterns of those edges connecting from the source node to the destination nodes are set as those in Figs. 15 and $16 ; 3$ ) transportation network with flow pattern planning but
TABLE I

EVACUATION RESULTS

\begin{tabular}{|c|c|c|c|c|}
\hline & \multicolumn{2}{|c|}{$1^{\text {st }}$ Category } & \multicolumn{2}{|c|}{$2^{\text {nd }}$ Category } \\
\hline & $\mathrm{TET}_{1}$ & $\mathrm{AET}_{1}$ & $\mathrm{TET}_{2}$ & $\mathrm{AET}_{2}$ \\
\hline Damaged network & 11 & 7.27 & 60 & 36.74 \\
\hline Predefined network & 9 & 6.80 & 46 & 30.27 \\
\hline $\begin{array}{l}\text { Flow pattern planning } \\
\text { without design }\end{array}$ & 7 & 5.40 & 28 & 20.18 \\
\hline $\begin{array}{l}\text { Integrated design and } \\
\text { flow pattern planning }\end{array}$ & 6 & 5.00 & 23 & 17.55 \\
\hline
\end{tabular}

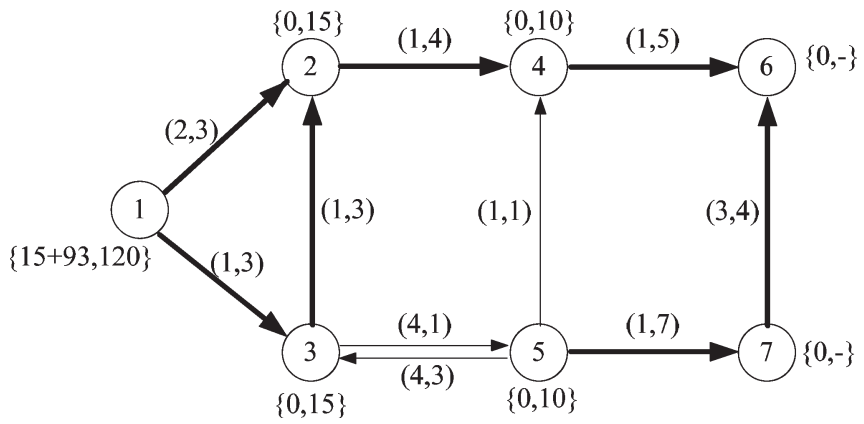

Fig. 17. Optimal flow patterns without design for the first category victim.

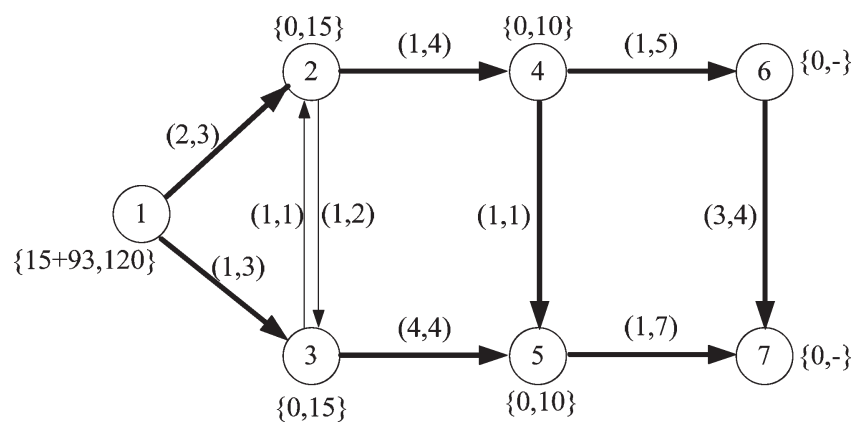

Fig. 18. Optimal flow patterns without design for the second category victim.

without design; and 4) transportation network with integrated design and flow pattern planning.

In our method of solving this problem, we have the following settings: 1) The swarm size in PSO $m=5$;2) the maximum iteration number in PSO $N G=10$; and 3) the inertia weight in PSO $\omega$ decreases linearly from 0.8 to 0.2 . The whole method is programmed using Java, and the experiment is performed on a computer with a dual $1.66-\mathrm{GHz} \mathrm{CPU}$ and a $1.5-\mathrm{GB}$ memory. The algorithm runs 50 times.

\section{B. Results and Discussion}

The evacuation performance of the four network settings is listed in Table I. The best flow patterns without design for the victims of the first and second categories are not unique, however; one of the best flow patterns for each category is shown, respectively, in Figs. 17 and 18. Under the optimal flowpattern planning without design, the total evacuation time of the first category is 7 ( $A E T=5.4)$, and the total evacuation time of the second category is $28(A E T=20.18)$. Therefore, for both 


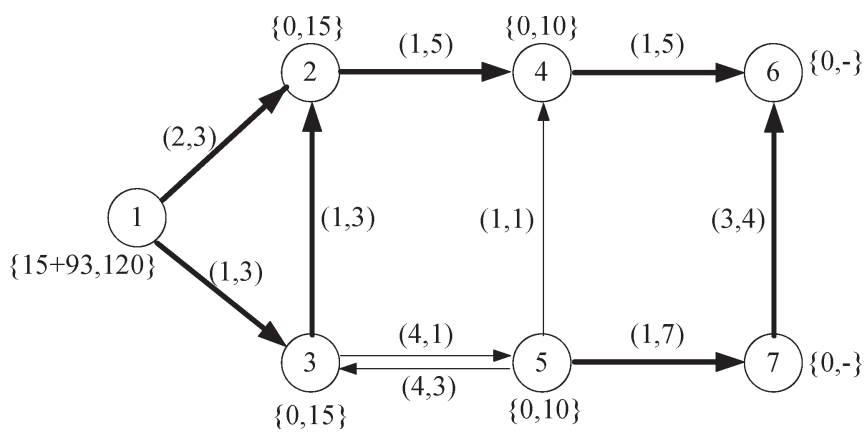

Fig. 19. Optimal flow patterns without design for the first category victim.

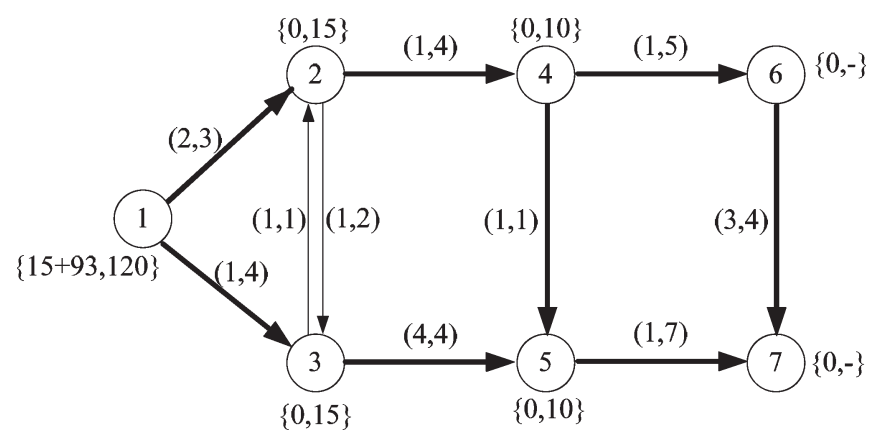

Fig. 20. Optimal flow patterns without design for the second category victim.

categories of victims, their evacuation performances are much better than those of the damaged and predefined networks.

For the integrated design and flow-pattern planning method, during the first period, the best design and construction of a new lane is found to add a lane $2 \rightarrow 4$. The best flow pattern under this design is also not unique; one of the best flow patterns is shown in Fig. 19. During the second period, the best design and construction of a new lane is found to add a lane $1 \rightarrow 3$. The best flow pattern under this design is not unique, which means that there are more than one flow pattern to achieve the minimum; one of the best flow patterns is shown in Fig. 20. We can see that under this integrated design and flowpattern planning, the total evacuation time of the first category is $6(A E T=5)$, and the total evacuation time of the second category is $23(A E T=17.55)$. Comparing with the results obtained from the damaged network, our proposed approach can reduce the evacuation time by more than $50 \%$. The total evacuation time of the proposed approach is about $20 \%$ less than that without incorporation of the road reconstruction. It should be noted that in an emergency case, the first-category victims may be seriously ill people, and the saving of time means increasing the probability of survival.

Next, we will give another illustration about the size of the search space for the given network. This example network has seven nodes. In the lower layer algorithm, the original network is converted to a TEN whose size is related to $T$ (the predetermined upper bound of the total travel time). We set $T$ to 100 in the experiment. Therefore, the TEN has 701 nodes. In the middle-layer algorithm, one solution represents a flow pattern of five roads, and the whole search space has 1024 solutions. In the upper layer algorithm, the whole search space has 18 solutions.
TABLE II

PSO PERformance With Different Parameters

\begin{tabular}{cccc}
\hline \hline $\begin{array}{c}\text { Example } \\
\text { network }\end{array}$ & Parameters & $\begin{array}{c}\text { Success } \\
\text { rate }\end{array}$ & $\begin{array}{c}\text { Running } \\
\text { time (s) }\end{array}$ \\
\hline 11 nodes, & $m=5, N G=10$ & $50 \%$ & 48.36 \\
15 arcs & $m=10, N G=10$ & $70 \%$ & 94.83 \\
& $m=10, N G=20$ & $100 \%$ & 92.67 \\
& $m=20, N G=5$ & $30 \%$ & 183.57 \\
& $m=20, N G=10$ & $30 \%$ & 151.826 \\
19 nodes, & $m=20, N G=20$ & $80 \%$ & 293.894 \\
30 arcs & $m=30, N G=5$ & $50 \%$ & 213.256 \\
& $m=30, N G=10$ & $70 \%$ & 427.938 \\
& $m=30, N G=20$ & $90 \%$ & 864.57 \\
\hline \hline
\end{tabular}

It is noted that in the three-layer method, the upper and lower layer algorithms are deterministic algorithms, and the middlelayer algorithm, i.e., PSO, is a random searching algorithm, which means that to test the stability and success rate of the method, we only need to test the PSO algorithm. We find the best parameters of the PSO algorithm, as stated in Section V-A, and under these settings, the success rate at which PSO achieves the global optimum in the given example is $100 \%$. The running time of the whole method to get design and planning results is about $30 \mathrm{~s}$.

To test the scalability issue of the method, we apply the method to the following networks: 1) 11 nodes and 15 two-way arcs and 2) 19 nodes and 30 two-way arcs. These two networks are the appropriate sizes in real-world applications. We test different parameters of PSO. The algorithm runs 50 times for each case.

The parameters of PSO are much related to the algorithm performance and running time. For example, when the parameters of PSO for the network with 11 nodes and 15 two-way arcs are set the same as those of the network with seven nodes and nine arcs, the success rate is $50 \%$. If we increase the swarm size $m$ to 10 and the maximum iteration number $N G$ to 20 , the success rate will increase to $100 \%$. However, the good performance results in the running time increasing up to four times the running time with the original parameter setting. The detail results of the PSO performance with different parameters are listed in Table II.

\section{RELATED WORK}

The resource planning in the transportation system in an emergency situation is an important research problem by itself, and it is called evacuation planning in the context of disaster management. The research about evacuation planning can be divided into three categories. The first category is used to treat an evacuation-planning problem at a policy level. Detailed reviews of the hurricane evacuation plans and policies from a transportation perspective are given in [19]-[21]. Their studies mainly focused on the current practices of the 
U.S., including the use of reverse flow operations, namely, contraflow operation and intelligent transportation systems. Urbina and Wolshon [19] and Wolshon et al. [20] summarized current evacuation-management policies, methods of information exchange, and decision-making criteria.

The second category is used to treat the evacuation-planning problem at a system level. Gwynne et al. [22], [23] concluded the methodologies for evacuation into three different approaches, namely, optimization, simulation, and risk assessment. Santos and Aguirre [24] presented a critical review of selected simulation models, including the following: 1) flow based; 2) cellular automata; 3) agent based; and 4) activity based. Kuligowski and Peacock [25] provided a comprehensive analysis of different features of existing evacuation systems based on the work of Gwynne et al. [22], [23].

The third category is used to treat an evacuation-planning problem at a model level. The models include optimization models and simulation models. Hamacher and Tjandra [26] presented a good review of mathematical models. Based on their research, Mamada et al. [27], [28] gave a further summary of the mathematical models. Among these studies, there are only a few that defined the evacuation problem as a multiobjective optimization problem. Hamacher and Tufekci [29] established a multiobjective model by considering the priorities of different evacuation areas, and the evacuees are moved from the higher priority regions to the lower priority regions. However, they did not consider the different demands of different categories of victims. Kostreva and Wiecek [30] proposed backward and forward dynamic programming algorithms to solve such a multiobjective problem. More recently, the system dynamic approach and the control theory are employed to model the evacuation problem. Simonovic and Ahmad [31] used the system dynamics approach to build an evacuation model for a flood emergency situation. Wadoo and Kachroo [32], [33] used the nonlinear feedback controller to represent evacuation dynamics in one and two dimensions.

Contraflow operation is an important measure for evacuation in practice, which is adapted in the hurricane evacuation plans by some U.S. states [21]. However, the contraflow operation in these plans is very simple, only considering that the roads linked with the hurricane source place are outbound and that the roads linked with the destination places are inbound. The quantitative analysis and research on the contraflow operation have only appeared over the past ten years. Dong and Xue [34], [35] applied artificial intelligence techniques to the contraflow plan. They considered one particular road, namely, a tunnel, in this case, in the regular situation and not a transportation network in the emergency situation, but their results indicated the potential foreground of using artificial intelligent method to solve the contraflow problem. Recently Kim et al. [1], [36] applied two heuristic methods and the SA algorithm to the contraflow configuration problem. They extended the contraflow operation to a transportation network and proved the efficiency of their algorithms. They did not consider the case of reversing two groups of lanes of different directions in one road at the same time. This is perhaps because they only considered the case where the capacity and the travel time along each of the lanes are the same. Another problem with their approach is that they preferred to use the simple heuristic algorithms to solve the contraflow problem and abandoned the intelligent-optimization algorithm in their latest paper [36]. The advantage of simple heuristic algorithms is the high efficiency in dealing with the high-dimension problem. However, simple heuristic algorithms lack the ability to get a global optimal solution. Intelligentoptimization algorithms have more potential to get a global optimal solution.

In our previous paper [37], we formulated the concept of the integrated road reconstruction and resource-replanning strategy and demonstrated its usefulness in emergency evacuation in a more realistic way. We considered replanning of the flow pattern using the contraflow method, but we considered a more general case than the studies of Kim and Shekhar [1] and Dong and Xue [34], [35]. In particular, the current literature considered three flow patterns in one road, and we considered four flow patterns in one road. Another difference between the literature and our previous paper [37] is that we used an intelligent-optimization algorithm, rather than a simple heuristic algorithm, to solve the problem. However, in our previous study, we have not considered the different demands of different categories of victims, nor have we considered the SSMD scenario. These two problems are discussed in this paper.

\section{CONClusion With Further Discussion}

The integrated road reconstruction and resource-replanning approach will add further value to the optimality of the process of evacuation of victims to safe destinations. The emergency evacuation problem is traditionally thought of as a resourceplanning problem. This may make sense for normal situations, but in disaster situations, construction of a new road or new lane (that is contraflow) and/or repair of a damaged road is common, which makes sense for putting these two activities (construction and planning) together. Our research concludes that the improvement with such an integrated approach is significant. If one new road is reconstructed, the evacuation time may be reduced by more than $50 \%$ of the evacuation time with the original resource operation on the damaged network and by about $20 \%$ of the evacuation time with the resource replanning only on the damaged transportation network. It is believed that such significance will also happen for similar service provider systems such as multicommodity supply chains.

At the computational aspect, our research concludes that the three-layer method is effective for the integrated design and planning problem. The upper layer algorithm is used to find an optimal design for a damaged transportation system; the PSO algorithm is used to find an optimal flow pattern for the given transportation system, and the lower layer algorithm, i.e., the P-MCMF algorithm, is used to find the best evacuation flow planning solution for victims of different categories. The lower layer algorithm provides an easy but effective solution for a kind of special MCMF problem that has not been considered in the literature. Further, this kind of MCMF problem has the following features: 1) There is more than one category of commodities that need to be shipped on a dynamic network (time-space network); 2) there are capacity constraints on the edges and nodes of the network; and 3) different category 
commodities have different priorities, and the commodity with a higher priority should be shipped earlier.

The computer running time of our proposed method mainly comes from the iterations of PSO, which has a much better potential ability to find the global optimum than the simple heuristic algorithms. It should be noted that, however, since PSO is a stochastic searching algorithm, it is hard to predict the time complexity of the proposed method because of the following three coupled factors: 1) the size of the transportation network; 2) the number of evacuees; and 3) the success rate. The third factor further shows that the longer the algorithm runs, the better the solutions that can be obtained, and therefore, one can terminate the algorithm at the time that the solution meets the evacuation demand. We have tested our method in the following three different networks, with a total of 108 evacuees: 1) seven nodes and nine two-way arcs; 2) 11 nodes and 15 twoway arcs; and 3) 19 nodes and 30 two-way arcs. The average running time for the 19 nodes network is about 213 s. This computation time is acceptable because it is much less than the setup time of the contraflow in practice, which is about 4-5 h.

As known, the evacuation time usually consists of the following three main time components [26]: 1) The time evacuees need to recognize a dangerous situation; 2) the time evacuees need to decide which course of action to take; and 3) the time evacuees need to move toward the safe area, which is also known as egress time. Since the behavioral and organizational factors are the main contributors to the first two time components, it is hard to analytically predict the duration of those time components. Therefore, most evacuation models consider the egress time only. The evacuation time so predicted is treated as the lower bound of the real evacuation time. This is also the approach in this paper. There is one time factor that has not been considered in our model, which is the time to construct a new road or repair a damaged road and time to reverse the lane. These times may be called setup time. In practice, the setup time may have a significant effect on the evacuation time. For example, in most U.S. states, estimates for the time required to fully implement contraflow after the decision has been made range from 4 to $12 \mathrm{~h}$. We will consider the setup time in our further study.

\section{REFERENCES}

[1] S. Kim and S. Shekhar, "Contraflow network reconfiguration for evacuation planning a summary of results," in Proc. GIS, Bremen, Germany, Nov. 4-5, 2005, pp. 250-259.

[2] I. L. Wang, "Shortest paths and multicommodity network flows," Ph.D. dissertation, Georgia Inst. Technol., Atlanta, GA, 2003.

[3] J. Farvolden, W. Powell, and I. Lustig, "A primal partitioning solution for the arc-chain formulation of a multicommodity network flow problem," Oper. Res., vol. 41, no. 4, pp. 669-693, Jul./Aug. 1993.

[4] A. Geoffrion, "Primal resource-directive approaches for optimizing nonlinear decomposable systems," Oper. Res., vol. 18, no. 3, pp. 375-403, May/Jun. 1970.

[5] F. Barahona and R. Anbil, "The volume algorithm: Producing primal solutions with a subgradient method," Math. Program., vol. 87, no. 3, pp. 385-399, 2000.

[6] G. Polak, "On a parametric shortest path problem from primal-dual multicommodity network optimization," Network, vol. 22, no. 3, pp. 283-295, 1992.

[7] L. Fleischer, "Approximating fractional multicommodity flow independent of the number of commodities," SIAM J. Discrete Math., vol. 13, no. 4 , pp. 505-520, Oct./Nov. 2000.
[8] J. L. Goffin, A. Haurie, and N. Philips, "Decomposition and nondifferentiable optimization with the projective algorithm," Manage. Sci., vol. 38, no. 2, pp. 284-302, Feb. 1992.

[9] H. Nagamochi, M. Fukushima, and T. Ibaraki, "Relaxation methods for the strictly convex multicommodity flow problem with capacity constraints on individual commodities," Network, vol. 20, no. 4, pp. 409-426, 1990.

[10] C. Barnhart, "Dual-ascent methods for large-scale multicommodity flow problems," Nav. Res. Logist., vol. 40, no. 3, pp. 305-324, 1993.

[11] R. C. Eberhart and J. Kennedy, "A new optimizer using particle swarm theory," in Proc. Int. Symp. Micro Mach. Human Sci., Nagoya, Japan, 1995, pp. 39-43.

[12] J. Kennedy and R. C. Eberhart, "Particle swarm optimization," in Proc. IEEE Int. Conf. Neural Netw., Perth, Australia, 1995, pp. 1942-1948.

[13] J. Kennedy, "The particle swarm: Social adaptation of knowledge," in Proc. IEEE Int. Conf. Evol. Comput., Indianapolis, IN, 1997, pp. 303-308.

[14] Y. Shi and R. C. Eberhart, "A modified particle swarm optimizer," in Proc. IEEE Int. Conf. Evol. Comput., Anchorage, AK, 1998, pp. 69-73.

[15] I. C. Trelea, "The particle swarm optimization algorithm: Convergence analysis and parameter selection," Inf. Process. Lett., vol. 85, no. 6, pp. 317-325, 2003

[16] J. W. Wang and D. W. Wang, "Particle swarm optimization with a leader and followers," Prog. Nat. Sci., vol. 18, no. 11, pp. 1437-1443, Nov. 2008.

[17] F. C. Wan, D. W. Wang, and Y. P. Li, "Particle swarm optimization of correlative product combinatorial introduction model," Chin. J. Control Decis., vol. 19, no. 5, pp. 520-524, 2004.

[18] J. Kennedy and R. C. Eberhart, "A discrete binary of the particle swarm algorithm," in Proc. IEEE Int. Conf. Syst., Man, Cybern., Orlando, FL, 1997, pp. 4104-4108.

[19] E. Urbina and B. Wolshon, "National review of hurricane evacuation plans and policies: A comparison and contrast of state practices," Transp. Res. Part A, vol. 37, no. 3, pp. 257-275, Mar. 2003.

[20] B. Wolshon, E. Urbina, M. Levitan, and C. Wilmot, "Review of policies and practices for hurricane evacuation-Part 2: Traffic operations, management, and control," Nat. Hazards Rev., vol. 6, no. 3, pp. 143-161, Aug. 2005.

[21] B. Wolshon, E. Urbina, C. Wilmot, and M. Levitan, "Review of policies and practices for hurricane evacuation-Part 1: Transportation planning, preparedness, and response," Nat. Hazards Rev., vol. 6, no. 3, pp. 129142, Aug. 2005.

[22] S. Gwynne, E. R. Galea, M. Owen, P. J. Lawrence, and L. Filippidis, "A review of the methodologies used in the computer simulation of evacuation from the built environment," Build. Environ., vol. 34, no. 6, pp. 741-749, Nov. 1999.

[23] S. Gwynne, E. R. Galea, M. Owen, P. J. Lawrence, and L. Filippidis, "A review of the methodologies used in evacuation modeling," Fire Mater. vol. 23, no. 6, pp. 383-388, 1999.

[24] G. Santos and B. E. Aguirre, "A critical review of emergency evacuation simulation models," in Proc. Conf. Building Occupant Movement During Fire Emergencies, Gaithersburg, MD, Jun. 9-10, 2004, pp. 27-52.

[25] E. D. Kuligowski and R. D. Peacock, "A review of building evacuation models," Nat. Inst. Standards Technol., Gaithersburg, MD, Tech. Note 1471, Jul. 2005.

[26] H. W. Hamacher and S. A. Tjandra, "Mathematical modelling of evacuation problems: A state of art," in Proc. Pedestrian Evac. Dyn., 2002, pp. 227-266.

[27] S. Mamada, K. Makino, and S. Fujishige, "Evacuation problems and dynamic network flows," in Proc. SICE Annu. Conf., Sapporo, Japan, Aug. 4-6, 2004, pp. 530-535.

[28] S. Mamada, K. Makino, T. Takabatake, and S. Fujishige, "The evacuation problem dynamic network flows and algorithms," in Proc. SICE Annu. Conf., Fukui, Japan, Aug. 4-6, 2003, pp. 2807-2811.

[29] H. W. Hamacher and S. Tufekci, "On the use of lexicographic min cost flows in evacuation modeling," Nav. Res. Logist., vol. 34, no. 4, pp. 487503, 1987.

[30] M. M. Kostreva and M. M. Wiecek, "Time dependency in multiple objective dynamic programming," J. Math. Anal. Appl., vol. 173, no. 1, pp. 289-307, Feb. 1993.

[31] S. P. Simonovic and S. Ahmad, "Computer-based model for flood evacuation emergency planning," Nat. Hazards Rev., vol. 34, no. 1, pp. 25-51, Jan. 2005

[32] S. A. Wadoo and P. Kachroo, "Feedback control design and stability analysis of one dimensional evacuation system," in Proc. IEEE ITSC, Toronto, ON, Canada, Sep. 17-20, 2006, pp. 618-623. 
[33] S. A. Wadoo and P. Kachroo, "Feedback control design and stability analysis of two dimensional evacuation system," in Proc. IEEE ITSC, Toronto, ON, Canada, Sep. 17-20, 2006, pp. 1108-1113.

[34] Z. Dong and D. Xue, "Intelligent scheduling of contraflow control operation using hierarchical pattern recognition and constrained optimization," Proc. IEEE Int. Conf. Syst., Man, Cybern._- "Computational Cybernetics and Simulation,” Orlando, FL, 1997, vol. 1, pp. 135-140.

[35] D. Xue and Z. Dong, "An intelligent contraflow control method for realtime optimal traffic scheduling using artificial neural network, fuzzy pattern recognition, and optimization," IEEE Trans. Control Syst. Technol., vol. 8, no. 1, pp. 183-191, Jan. 2000.

[36] S. Kim, S. Shekhar, and M. Min, "Contraflow transportation network reconfiguration for evacuation route planning," IEEE Trans. Knowl. Data Eng., vol. 20, no. 8, pp. 1-15, Aug. 2008.

[37] J. W. Wang, W. J. Zhang, and D. W. Wang, "An integrated design and planning of networked service systems for high resilience-In the case of public transportation systems," Eur. J. Oper. Res., to be published.

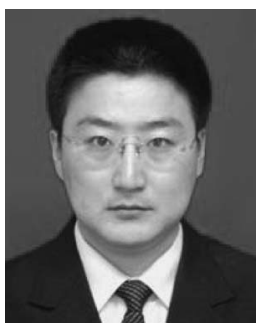

J. W. Wang received the Ph.D. degree in systems engineering from the Northeastern University, Shenyang, China, in 2006.

He has been a lecturer with the Institute of Systems Engineering, Northeastern University, since 2006. He has also been a research associate with the Department of Mechanical Engineering, the University of Saskatchewan, Saskatoon, SK, Canada, since 2007. His current research interests are modeling and optimization for resilience engineering of complex human-machine networked systems.

Dr. Wang has published several refereed technical papers and refereed conference papers and has co-authored a book on optimization algorithms.

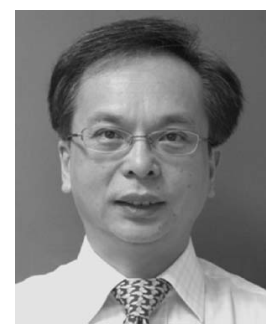

W. H. Ip received the M.Sc. degree from Cranfield University, Bedfordshire, U.K., in 1983, the M.B.A. degree from Brunel University, Uxbridge, U.K., in 1989, and the $\mathrm{Ph} . \mathrm{D}$. degree in manufacturing engineering from Loughborough University, Loughborough, U.K., in 1993.

$\mathrm{He}$ is an Associate Professor with the Department of Industrial and Systems Engineering, Hong Kong Polytechnic University, Kowloon, Hong Kong. He is the author of more than 100 published international journals and conference articles in the areas of production management, enterprise resource plannings, supply chains, and logistics systems. His research interests include the modeling and application of operations research and artificial intelligence techniques.

Dr. Ip is a member of the Institution of Mechanical Engineers, the Institution of Electrical Engineers (U.K.), and the Institution of Hong Kong Engineers.

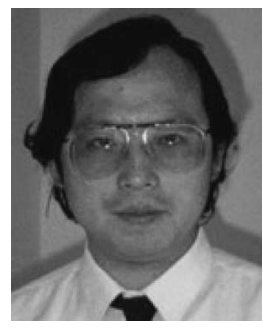

W. J. Zhang received the Ph.D. degree in design theory and computer-aided design from Delft University of Technology, Delft, The Netherlands, in 1994.

He is currently a Professor with the Department of Mechanical Engineering and the Chair of the Division of Biomedical Engineering, University of Saskatchewan, Saskatoon, SK, Canada. His current research interests include informatics, design, modeling and control of micromotion systems, modeling and management of large complex systems, and socio-tech and physical-biological system interaction and human behaviors. $\mathrm{He}$ is the author of over 135 technical papers published in peer-refereed journals.

Dr. Zhang is a member of the American Society of Mechanical Engineers, a Senior Member of the Society of Manufacturing Engineers, a member of The Information Resources Management Association, and a member of The International Society for Optical Engineering. 